\title{
Does IRISIN Have a BRITE Future as a Therapeutic Agent in Humans?
}

\author{
Brian A. Irving • Christopher D. Still • \\ George Argyropoulos
}

Published online: 2 February 2014

(C) The Author(s) 2014. This article is published with open access at Springerlink.com

\begin{abstract}
The epidemic of obesity has contributed to the rapid rise in comorbid conditions such as cardiovascular disease, type 2 diabetes, sleep apnea, and hypertension among others. Therefore, there is a critical need to develop therapeutic strategies to reduce the prevalence of the disease. Skeletal muscle cells secrete signaling cytokines/peptides (referred to as myokines) that act in autocrine, paracrine, and endocrine fashion. Myokines have been hypothesized to contribute to the immediate and chronic benefits of exercise and may thus serve as attractive therapeutic agents for the treatment of obesity. The recent discovery of the irisin, a proposed myokine, has gained much attention over the last two years as a potential therapeutic agent. Preliminary studies demonstrated that irisin has the potential to induce "browning" of white adipocytes in mice. If these findings in mice could be translated to humans, irisin could be a potential therapeutic agent for the treatment of obesity. Limitations with the available antibodies, however, have raised concerns regarding the detectability of irisin in circulation. Moreover, the gene encoding irisin, FNDC5, is expressed robustly not only in muscle but also in various white adipose tissues (WAT) in humans, raising the possibility for increased thermogenesis
\end{abstract}

\section{B. A. Irving $\cdot$ C. D. Still $•$ G. Argyropoulos \\ Department of Gastroenterology, Geisinger Medical Center, Danville, PA, USA}

B. A. Irving · C. D. Still • G. Argyropoulos

Geisinger Obesity Institute, Geisinger Medical Center, Danville, PA, USA

B. A. Irving $\cdot$ G. Argyropoulos

Weis Center for Research, Geisinger Medical Center, Danville, PA, USA

\section{B. A. Irving $(\bowtie)$}

Geisinger Obesity Institute, Geisinger Health System, 100 N.

Academy Ave, Danville, PA 17868, USA

e-mail: bairving@geisinger.edu through autocrine mechanisms. Here we will discuss the browning of WAT, the discovery of irisin, and its potential role in improving metabolic health in humans.

Keywords Adaptive thermogenesis · Uncoupling · Physical activity $\cdot$ Weight loss $\cdot$ Myokines $\cdot$ Adipokines $\cdot$ FNDC5 . Metabolism · Human · Mice · Brown adipose tissue - White adipose tissue $\cdot$ Beige adipose tissue $\cdot$ Skeletal muscle . Mitochondria $\cdot$ UCP1 $\cdot$ Irisin $\cdot$ Brite adipocytes

\section{Introduction}

Obesity has rapidly become a worldwide epidemic. In parallel, the prevalence of obesity-related comorbid conditions has also escalated, including insulin resistance, metabolic syndrome, type 2 diabetes, hypertension, chronic kidney disease, cardiovascular disease, heart failure, cancer, and dementia [1-3]. As expected, a recent meta-analysis from the US Centers for Disease Control and Prevention confirms that obesity is associated with increased all-cause mortality [4]. The rapid increase in obesity and obesity-related comorbid conditions has coincided with the rapidly changing landscape of our obesogenic environment [5]. In particular, it has coincided with the systemic reductions in total daily physical activity as well as reductions in vigorous physical activity [5, 6]. Exercise has long been recognized for its pluripotent effects on body composition [7, 8], metabolic health [9], cardiovascular disease $[10,11]$ and mental health [12]. The underlying mechanism(s) for the clinical benefits of exercise remains to be fully elucidated. Over the past decade, it has become increasingly recognized that skeletal muscle cells secrete signaling cytokines/peptides that act in autocrine, paracrine, and endocrine fashion in response to skeletal muscle contraction (e.g., exercise) [13]. The secreted cytokines/peptides, referred to as myokines, have been hypothesized to contribute to the 
immediate and chronic benefits of exercise [13]. The recent discovery of irisin by Bostrom et al. [14••], a putative exercise induced myokine, that is credited for improving metabolic health by its ability to brown white adipose tissue (WAT) in mice has received considerable attention over the last two years. Although it remains to be determined whether irisin has the ability to brown WAT and improve metabolic health in humans, it represents a potentially attractive therapeutic agent for treating obesity and metabolic disease in humans. Here we will briefly discuss the browning of WAT, the discovery of irisin, and the potential role that irisin may play in browning WAT and improving metabolic health in humans.

\section{Browning of White Adipocytes}

Classically, adipose tissue is characterized as either WAT or brown adipose tissue (BAT). Adipocytes from WAT serve as the primary site for lipid storage; whereas adipocytes from BAT are highly specialized cells designed to produce heat through uncoupled respiration that leads to concomitant dissipation of energy [15]. The physical, metabolic, and regulatory characteristics of WAT and BAT have been extensively reviewed elsewhere [16-21]. In brief, adipocytes from WAT have a unilocular lipid droplet, few mitochondria, and a relatively low metabolic rate [21]. In contrast, adipocytes from BAT have multilocular lipid droplets, many mitochondria, and a relatively high metabolic rate [21]. The relatively high metabolic rate observed in BAT compared to WAT is due to the presence of uncoupling protein 1 (UCP1), which is negligibly expressed in WAT [21].

The presence of brown adipocytes in WAT has been known for many years. Young et al. [22] were the first to report the presence of brown adipocytes in WAT of female BALB/c mice following cold acclimatization. Subsequently, brown adipocytes were identified in multiple WAT fat pads in rats [23]. Enrichment and activation of BAT represents an attractive therapeutic strategy to combat obesity and metabolic disease. The presence of UCP1 positive cells in WAT can also be pharmacologically enriched by $\beta$-adrenergic stimuli [23-25] as well as PPAR $\gamma$ agonist [26-28]. Recent evidence has demonstrated that the brown adipocytes (i.e., UCP1 positive cells) found in WAT are actually a distinct sub-population of white adipocytes (referred to as brown-in-white (brite) or beige adipocytes) $[15,26]$. Co-culture experiments demonstrated that beige/brite adipocytes treated with rosiglitazone (a PPAR $\gamma$ agonist) can be induced to differentiate into adipocytes with thermogenic potential in the absence of classical brown adipocyte-specific markers (e.g., Zic2, Lhx8, Meox3, and PRDM16) [26]. In addition, these beige/brite adipocytes are also characterized as having the white adipocyte-specific marker Hoxc9 while lacking the white adipocyte-specific marker tcf21 [26]. Taken together, these results indicate that the beige/brite adipocytes are truly a distinct subtype of white adipocytes with potentially hidden capacity for higher metabolic rate [26]. In 2012, Wu et al. [15] confirmed that the beige/brite adipocytes found in WAT depots in mice and humans are in fact distinct from classical brown adipocytes that are more abundant in mice as a specialized depot $[19,29]$. Specifically, for the first time they demonstrated that the beige/brite adipocytes emerge from non-myf-5 progenitor cells, in contrast to brown adipocytes, which are derived from myf-5 positive progenitor cells [15]. The exquisite regulation of the "browning" of white adipocytes in response to environmental, hormonal, and metabolic stimuli is quite remarkable. Excellent reviews of the development and regulatory control of beige/brite adipocytes have recently been published [19, 29]. Similar to BAT, enrichment and activation of beige/brite adipocytes represents an attractive therapeutic strategy to combat obesity and metabolic disease. The recent discovery of irisin and its potential to induce "browning" of white adipocytes has gained much attention over the last two years [14••], which will be discussed in detail below.

\section{Discovery of Irisin}

It is well accepted that exercise is the corner stone of a healthy lifestyle and the frontline defense for primary and secondary prevention of many metabolic and cardiovascular diseases. Chronic endurance training has also been shown to induce skeletal muscle mitochondrial biogenesis [30,31], which is regulated by the expression and activity of PPAR- $\gamma$ coactivator-1 $\alpha$ PGC1- $\alpha$ [31-33]. Indeed, the canonical PGC1- $\alpha$ (i.e., PGC1- $\alpha 1$ [34]) has been shown to be the master regulator of mitochondrial biogenesis in multiple tissues [32, 33]. Likewise, skeletal muscle over-expression of PGC1- $\alpha 1$ mimics many of the protective effects of exercise on multiple tissues $[35,36]$. Interestingly, the newly identified splice variant of PGC1- $\alpha, \mathrm{PGC} 1-\alpha 4$, has also been shown to induce skeletal muscle hypertrophy when transgenically overexpressed in mice consistent with a resistance-trained phenotype [34]. As such, efforts were made to identify the mechanism by which the expression and activity of PGC1- $\alpha$ in skeletal muscle affects other tissues. These studies were conducted under the backdrop of recent interest in skeletal muscle secreted signaling peptides referred to as myokines [37]. Could the increased expression of PGC1- $\alpha$ in skeletal muscle result in the secretion of some known or unknown set of myokines that affect the function of other tissues?

Bostrom et al. [14*0] first examined the WAT depots of muscle specific PGC1- $\alpha$ transgenic mice to determine if there were molecular and/or metabolic differences in WAT compared to their wild-type littermates. They demonstrated that the inguinal WAT (subcutaneous WAT) consisted of a population of beige/brite adipocytes that had increased levels of 
UCP1 and Cidea [14••] in muscle specific PGC1- $\alpha$ transgenic mice compared to their wild-type littermates. Next, they demonstrated that wheel running and swimming substantially induced the expression of UCP1 in inguinal WAT ( 25 fold, $\sim 65$-fold, respectively) [14••]. Taken together, these findings indicate that increased expression of PGC1- $\alpha$ in murine skeletal muscle induces browning of inguinal WAT, similar to what is observed through traditional exercise regimens. To address whether this browning of the subcutaneous WAT was due directly to muscle-fat cell signaling (e.g., myokine stimulated browning of WAT) they next cultured primary murine subcutaneous adipocytes with conditioned media from PGC1- $\alpha$ over expressing murine myocytes. Again, they found that the conditioned media treated murine subcutaneous adipocytes had increased expression brown-fat-specific genes (e.g., UCP1 and Cidea) [37]. These findings suggest that PGC1- $\alpha$ overexpressing murine myocytes secrete one or more myokines capable of inducing a thermogenic program in murine subcutaneous adipocytes.

Bostrom et al. [14••] used a combination of gene array technology and advanced bioinformatic algorithms to predict potential proteins that could be secreted by skeletal muscle and induce browning of WAT. Fibronectin type III domaincontaining 5 (FNDC5) was one of five target genes of PGC1- $\alpha$ which could be secreted [14••]. Importantly, FNDC5 expression was increased in muscle from exercise trained mice as well as humans [14••]. Next, they demonstrated that the treatment of primary subcutaneous adipocytes during differentiation with recombinant-FNDC5 increased the expression of BAT genes (UCP1, Elov13, Cox7a, and Otop1) [14••]. Moreover, recombinant-FNDC5 treated UCP1-positive cells also developed multilocular lipid droplets and increased mitochondrial content [14••]. Importantly, high-resolution respirometry experiments revealed that the recombinant-FNDC5 treated UCP1-positive cells had increased oxygen consumption, particularly with respect to uncoupled respiration [14••]. Taken together, the authors concluded that these findings indicate that FNDC5 induces UCP1-positive cells to develop the beige/brite phenotype in mice [14••]. However, it should be noted that the recombinant-FNDC5 protein used in these

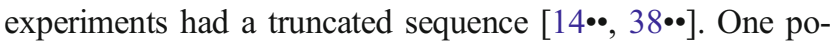
tential mechanism by which FNDC5 induces browning of subcutaneous adipocytes is through increasing the expression of PPAR- $\alpha$ [14••]. Indeed, treatment of primary subcutaneous adipocytes with a PPAR- $\alpha$ antagonist attenuated the browning effect of the recombinant-FNDC5 [14••].

Before the protein product of FNDC5 was termed "irisin" [14••], it had already been discovered by two independent groups and assigned the gene aliases "PeP" [39] and "frcp2" [40]. PeP and frcp 2 were both found to be expressed in skeletal muscle, heart, and brain of adult mice [39, 40]. It should be noted, however, that PeP and frcp2 expression in adipose tissue was not assessed [39, 40]. Bostrom et al. [14••] were the first to recognize and suggest that although the fulllength FNDC5 is a trans-membrane protein, its extracellular N-terminal portion of FNDC5 could potentially be cleaved by a yet to be identified protease. Identification of the FNDC5 fragment was initially determined by antibody binding, which was confirmed by mass spectrometry [14॰•]. Their analysis also revealed that the secreted form of FNDC5 was highly homologous between mouse and humans [14••]. They named this newly identified signaling peptide (myokine) irisin after the messenger goddess of ancient Hellenic mythology, Iris [14••]. Another potential mechanism that has been suggested for observing N-terminal (extracellular) fragments of FNDC5 (e.g., irisin) in cell culture media and/or plasma in vivo is through shedding of the extracellular fraction [38••].

Using an antibody against FNDC5 Bostrom et al. [14••] were able to reduce the browning effect of media conditioned by skeletal muscle PGC1- $\alpha$ overexpressing murine myocytes on primary subcutaneous fat. Of note, the antibody used in these experiments was targeted at the c-terminus of FNDC5, which should not be present in irisin. They also indicated that irisin was present in both mouse and human plasma [14••]. Moreover, they demonstrate that plasma irisin concentrations were elevated in mice and older humans after short-term exercise training [14••]. Using adenoviral delivery of FNDC5 to the liver they were also able to increase plasma concentration of irisin which led to the browning of subcutaneous WAT while protecting against diet induced obesity and insulinresistance $[14 \bullet \bullet$. The increased circulating levels of irisin were also associated with increased expression of mitochondrial genes in the subcutaneous WAT and a concomitant increase in oxygen consumption. Taken together these data indicate that irisin, which is secreted from active (murine) skeletal muscle has the potential to protect against obesity and insulin resistance. However, it remains to be demonstrated that irisin is secreted from human skeletal muscle, and if not so to determine whether the potential action of FNDC5 and/or irisin is derived from non-skeletal muscle tissue.

\section{Does Irisin affect Human White Adipocytes}

Several manuscripts have demonstrated that irisin enhances the "browning" of white adipocytes in mice [14••, 15], particularly in white adipocytes that highly express CD137 [15]. However, recent evidence has begun to question the physiological relevance of irisin in humans [41••, 42••]. Can the results obtained in mice be readily translated to humans? Specifically, is irisin synthesized and secreted from active human skeletal muscle? Is skeletal muscle the primary source of irisin and/or FNDC5? Can irisin induce browning of white adipocytes in humans?

As previously discussed, Bostrom et al. [14••] reported that 10 weeks of combined endurance plus resistance exercise 
training increases circulating levels of irisin in older adults approximately two-fold, which was proportional to the increase in skeletal muscle mRNA expression of FNDC5. However, Timmons et al. [42••] demonstrated that neither endurance training nor resistance training increases skeletal muscle FNDC5 mRNA expression in healthy adults. Nevertheless, they did report that FNDC5 expression was elevated in a subset of exercise trained older adults but not younger adults compared to their sedentary counterparts, which is an inter-

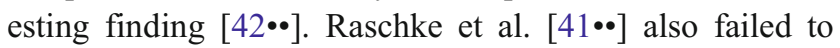
demonstrate an increase in FNDC5 expression using an in vitro model of endurance training (electrical pulse stimulation) in human myotubes as well as in response to aerobic interval training and strength training in sedentary males. Results of a recently published randomized clinical trial of $(n=102)$ middle aged (30-60 years old) participants demonstrated that neither endurance training nor resistance training increase circulating irisin concentrations after 26 weeks of training compared to controls [43•]. An important observation from this study was that irisin is prone to storage-related degradation [43•]. Therefore, time related changes in circulating irisin concentrations in the absence of timed-matched controls should be interpreted with caution. In another study, it was demonstrated that an acute bout of endurance exercise, chronic endurance exercise, and chronic endurance combined with resistance exercise provide conflicting results with respect to skeletal muscle PGC1- $\alpha$ expression, FNDC5 expression, and circulating irisin [44•]. Twelve weeks of exercise training has also been reported to have little to no effect on genes expression in subcutaneous WAT for genes associated with browning of WAT (e.g., UCP1, PRDM16, TBX1, TMEM26, or CD137), despite significant increases in skeletal muscle FNDC5 [45••]. Taken together, the results of Timmons et al. [42•], Raschke et al. [41••], Hacksteden et al. [43•], Pekkela et al. [44•], and Norheim et al. [45••] raise significant concerns regarding the muscle-specific effects of exercise training on the stimulation of irisin in humans.

\section{Putative Effects of FNDC5/Irisin on Browning of White Adipocytes}
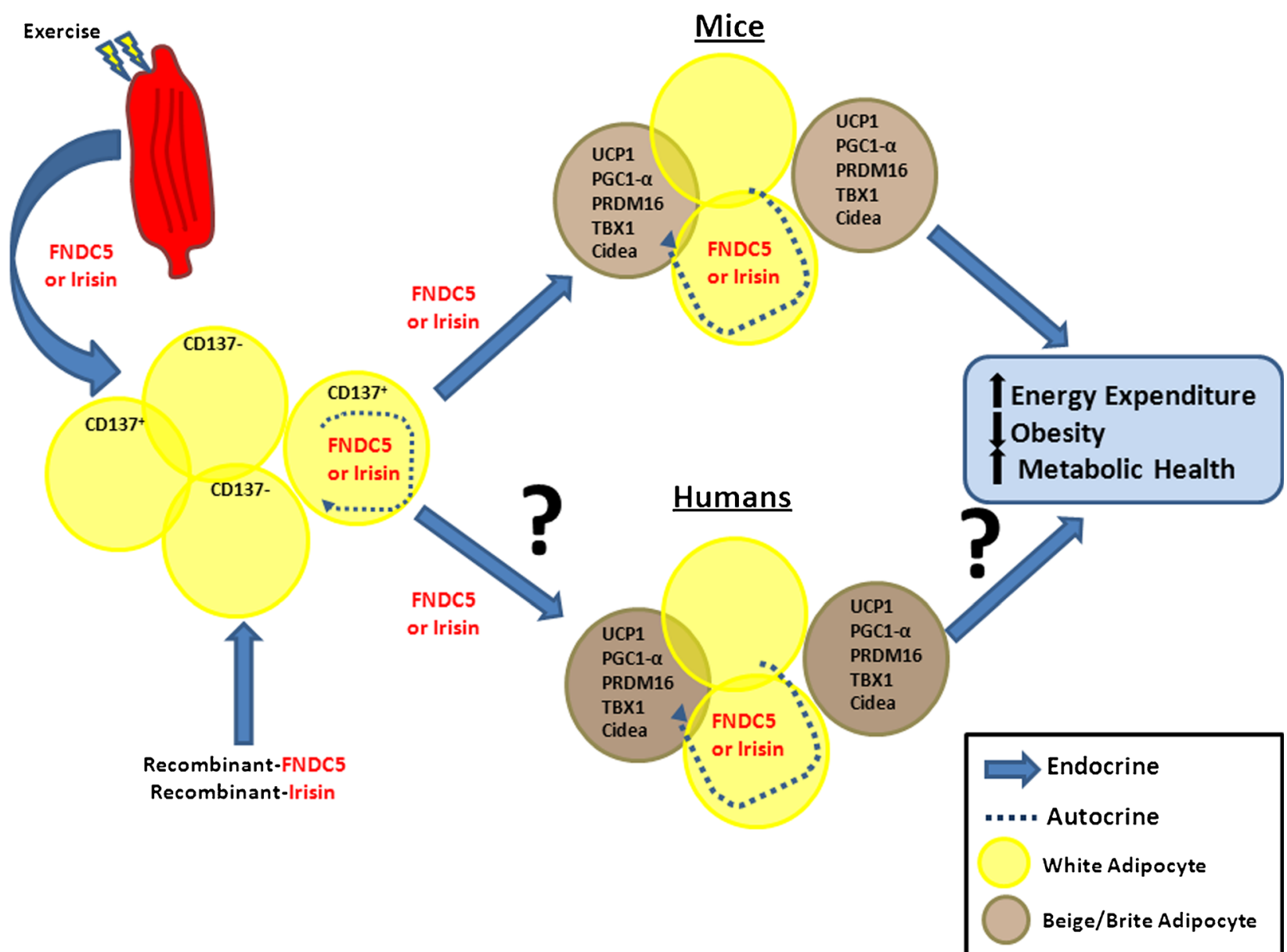

Fig. 1 Putative effects of FNDC5/irisin on browning of white adipocytes and improvements in energy expenditure, obesity, and metabolic health in mice and humans 
The human gene sequence of FNDC5 is also raising concerns regarding the translated product [46]. Although the FNDC5 gene sequence is highly conserved across species [14••], the human FNDC5 gene has a variation in its start codon that could essentially affect its translation [46]. Specifically, the translational initiation "ATG" codon is mutated to "ATA" resulting in substantial reduction in the translational efficiency of FNDC5 and leading to the translation of only $1 \%$ of full-length FNDC5 as reported [41 • $]$. Furthermore, there is a truncated isoform of FNDC5 that is translated by a downstream "ATG" start codon that is lacking the signaling peptide $[41 \bullet \bullet]$. Consistent with this later finding, on September 5th 2012, the UniProt database was annotated to include a second, truncated, protein sequence for FNDC5 [41••]. Raschke et al. [41••] further demonstrated that neither recombinant-irisin nor recombinant-FNDC5 induces "browning" of human pre-adipocytes. In contrast, they demonstrated that BMP7 (i.e., the positive control) resulted in an activation of the genes regulating the "browning" of human preadipocytes characterized by elevations in PPAR $\gamma, \mathrm{UCP} 1$, PGC-1 $\beta$, as well as an elevation in mitochondrial protein content $[41 \bullet \cdot$. Taken together, these findings indicate that the ability of endogenous irisin to stimulate "browning" of white adipocytes in humans remains to be proven.

Surprisingly, little information is known about the expression and potential secretion of FNDC5/irisin from adipose tissue itself. A few recent papers, however, highlight the presence of FNDC5 in rat and human WAT [47, 48••, 49]. Using gene-array technology our research team recently demonstrated that FNDC5 is highly expressed in visceral adipose tissue, epigastric adipose tissue, and to a lesser extent subcutaneous adipose tissue of severely obese patients undergoing bariatric surgery [48••]. Likewise, PGC1- $\alpha$ was also abundantly expressed in these adipose tissue depots [48••]. Taken together, it appears that human WAT has some of the key components necessary for FNDC5-induced browning WAT in an autocrine fashion in humans. In another study, it was demonstrated by real-time PCR that FNDC5 gene expression was reduced in obese as well as patients with type 2 diabetes [49]. Moreover, FNDC5 gene expression in visceral and subcutaneous WAT was positively associated with brown adipose tissue markers (PRDM16 and UCP1) in humans [49]. Future investigations are warranted to examine the potential role of FNDC5 to induce browning of WAT in humans via autocrine mechanisms. However, it has been reported that the expression of FNDC5 in WAT is less than five percent of that observed in skeletal muscle in humans [49, 50]. RocaRivada et al. [47] recently reported that WAT explants secrete FNDC5/irisin, which is increased after 1 week of exercise in rats [47]. Interestingly, however, the secretion of FNDC5/ irisin from the WAT explants was reduced after 3 weeks of exercise training [47]. The secretion of FNDC5/irisin was based on quantification of the $25 \mathrm{kDa}$ band (predicted MW of irisin $\sim 12 \mathrm{kDa}$ ), which was detected both by the Abcam and the Phoenix antibodies [47]. Moreover, the Abcam FNDC5 antibody recognizes the C-terminus of FNDC5 that is not supposed to be part of irisin [38*0]. It should also be noted, the Abcam and the Phoenix antibodies do not share any sequence overlap [38••]. In summary, it appears that human WAT has some of the key components necessary for FNDC5 to act in an autocrine fashion to brown WAT in humans. However, future studies are needed using validated antibodies to determine whether human WAT secretes full-length FNDC5 and/or irisin. Likewise, experimental evidence demonstrating the ability of FNDC5/irisin to brown human WAT resulting in increased UCP1 expression and thermogenesis regardless of its source is currently lacking.

\section{Conclusion}

The recent discovery of irisin has garnered much attention as a potential therapeutic agent for the treatment of obesity and its comorbid conditions. Figure 1 presents the putative effects of irisin on the browning of murine and human white adipocytes. Preliminary studies have indicated that recombinant FNDC5 and/or irisin can induce browning of murine WAT. However, there remains a dearth of evidence to indicate that recombinant FNDC5 and/or irisin can induce browning of human WAT. Moreover, recent studies in humans also indicate that neither acute nor chronic exercise consistently results in increased expression of endogenous FNDC5 and/or increased circulating concentrations of FNDC5/irisin in humans. These later findings may be due in part to the lack of available antibodies that are validated to detect FNDC5/irisin. In addition, care should be taken when extrapolating data derived from mouse studies to human physiology given the significant differences in the abundance of brown fat between humans and mice. Finally, the expression of FNDC5 in human WAT opens the door to the possibility that FNDC5 acts in an autocrine fashion to brown WAT in humans.

Acknowledgments This research was supported by research funds from the Geisinger Health System and the National Institutes of Health (grant P30 DK072488 to BAI, CDS, and GSG).

Funding This research was supported by funds from the Geisinger Health System and the National Institutes of Health (grant P30 DK072488).

\section{Compliance with Ethics Guidelines}

Conflict of Interest Brian A. Irving, Christopher D. Still, and George Argyropoulos declare that they have no conflict of interest.

Human and Animal Rights and Informed Consent This article does not contain any studies with human or animal subjects performed by any of the authors. 
Open Access This article is distributed under the terms of the Creative Commons Attribution License which permits any use, distribution, and reproduction in any medium, provided the original author(s) and the source are credited.

\section{References}

Papers of particular interest, published recently, have been highlighted as:

- Of importance

-. Of major importance

1. Grundy SM, Brewer Jr HB, Cleeman JI, Smith Jr SC, Lenfant C, American Heart A, et al. Definition of metabolic syndrome: report of the National Heart, Lung, and Blood Institute/American Heart Association conference on scientific issues related to definition. Circulation. 2004;109(3):433-8. doi:10.1161/01.CIR. 0000111245.75752.C6.

2. Mokdad AH, Ford ES, Bowman BA, Dietz WH, Vinicor F, Bales VS, et al. Prevalence of obesity, diabetes, and obesity-related health risk factors, 2001. JAMA. 2003;289(1):76-9.

3. Lloyd-Jones D, Adams R, Carnethon M, De Simone G, Ferguson TB, Flegal K, et al. Heart disease and stroke statistics-2009 update: a report from the American Heart Association Statistics Committee and Stroke Statistics Subcommittee. Circulation. 2009;119(3):e21e181. doi:10.1161/CIRCULATIONAHA.108.191261.

4. Flegal KM, Kit BK, Orpana H, Graubard BI. Association of allcause mortality with overweight and obesity using standard body mass index categories: a systematic review and meta-analysis. JAMA. 2013;309(1):71-82. doi:10.1001/jama.2012.113905.

5. Chaput JP, Klingenberg L, Astrup A, Sjodin AM. Modern sedentary activities promote overconsumption of food in our current obesogenic environment. Obes Rev: Off J Int Assoc Study Obes. 2011;12(5):e12-20. doi:10.1111/j.1467-789X.2010.00772.x.

6. Church TS, Thomas DM, Tudor-Locke C, Katzmarzyk PT, Earnest CP, Rodarte RQ, et al. Trends over 5 decades in U.S. occupationrelated physical activity and their associations with obesity. PloS one. 2011;6(5):e19657.

7. Irving BA, Davis CK, Brock DW, Weltman JY, Swift D, Barrett EJ, et al. Effect of exercise training intensity on abdominal visceral fat and body composition. Med Sci Sports Exerc. 2008;40(11):186372. doi:10.1249/MSS.0b013e3181801d40.

8. Irwin ML, Yasui Y, Ulrich CM, Bowen D, Rudolph RE, Schwartz $\mathrm{RS}$, et al. Effect of exercise on total and intra-abdominal body fat in postmenopausal women: a randomized controlled trial. JAMA. 2003;289(3):323-30.

9. Johnson JL, Slentz CA, Houmard JA, Samsa GP, Duscha BD, Aiken LB, et al. Exercise training amount and intensity effects on metabolic syndrome (from Studies of a Targeted Risk Reduction Intervention through Defined Exercise). Am J Cardiol. 2007;100(12):1759-66. doi:10.1016/j.amjcard.2007.07.027.

10. Thompson PD, Buchner D, Pina IL, Balady GJ, Williams MA, Marcus BH, et al. Exercise and physical activity in the prevention and treatment of atherosclerotic cardiovascular disease: a statement from the Council on Clinical Cardiology (Subcommittee on Exercise, Rehabilitation, and Prevention) and the Council on Nutrition, Physical Activity, and Metabolism (Subcommittee on Physical Activity). Circulation. 2003;107(24):3109-16. doi:10. 1161/01.CIR.0000075572.40158.77.

11. Manson JE, Hu FB, Rich-Edwards JW, Colditz GA, Stampfer MJ, Willett WC, et al. A prospective study of walking as compared with vigorous exercise in the prevention of coronary heart disease in women. N Engl J Med. 1999;341(9):650-8. doi:10.1056/ NEJM199908263410904.

12. Weuve J, Kang JH, Manson JE, Breteler MM, Ware JH, Grodstein F. Physical activity, including walking, and cognitive function in older women. JAMA. 2004;292(12):1454-61. doi:10.1001/jama. 292.12.1454.

13. Pedersen BK, Febbraio MA. Muscles, exercise and obesity: skeletal muscle as a secretory organ. Nat Rev Endocrinol. 2012;8(8):45765. doi:10.1038/nrendo.2012.49.

14.• Bostrom P, Wu J, Jedrychowski MP, Korde A, Ye L, Lo JC, et al. A PGC1-alpha-dependent myokine that drives brown-fat-like development of white fat and thermogenesis. Nature. 2012;481(7382): 463-8. doi:10.1038/nature10777. Paper describes the initial dicovery of irisin. The authors demonstrate the FNDC5 / irisin is secreted from murine muscle cells, particularly during exercise. Moreover, they demonstrate that exercise trianing results in browning of subcutaneous white adipose tissue in mice. Finally, the paper describes the ability of recombinant-FNDC5 to brown murine white adipocytes.

15. Wu J, Bostrom P, Sparks LM, Ye L, Choi JH, Giang AH, et al. Beige adipocytes are a distinct type of thermogenic fat cell in mouse and human. Cell. 2012;150(2):366-76. doi:10.1016/j.cell.2012.05.016.

16. Giralt M, Villarroya F. White, brown, beige/brite: different adipose cells for different functions? Endocrinology. 2013;154(9):29923000. doi:10.1210/en.2013-1403.

17. Cannon B, Nedergaard J. Brown adipose tissue: function and physiological significance. Physiol Rev. 2004;84(1):277-359. doi: 10.1152/physrev.00015.2003.

18. Cinti S. The adipose organ at a glance. Dis Models Mech. 2012;5(5):588-94. doi:10.1242/dmm.009662.

19. Bartelt A, Heeren J. Adipose tissue browning and metabolic health. Endocrinology: Nature reviews; 2013.

20. Gesta S, Tseng YH, Kahn CR. Developmental origin of fat: tracking obesity to its source. Cell. 2007;131(2):242-56. doi:10.1016/j. cell.2007.10.004.

21. Spiegelman BM. Banting Lecture 2012: regulation of adipogenesis: toward new therapeutics for metabolic disease. Diabetes. 2013;62(6):1774-82. doi:10.2337/db12-1665.

22. Young P, Arch JR, Ashwell M. Brown adipose tissue in the parametrial fat pad of the mouse. FEBS Lett. 1984;167(1):10-4.

23. Cousin B, Cinti S, Morroni M, Raimbault S, Ricquier D, Penicaud $\mathrm{L}$, et al. Occurrence of brown adipocytes in rat white adipose tissue: molecular and morphological characterization. J Cell Sci. 1992;103(Pt 4):931-42.

24. Seale P, Bjork B, Yang W, Kajimura S, Chin S, Kuang S, et al. PRDM16 controls a brown fat/skeletal muscle switch. Nature. 2008;454(7207):961-7. doi:10.1038/nature07182.

25. Guerra C, Koza RA, Yamashita H, Walsh K, Kozak LP. Emergence of brown adipocytes in white fat in mice is under genetic control. Effects on body weight and adiposity. J Clin Invest. 1998;102(2): 412-20. doi:10.1172/JCI3155.

26. Petrovic N, Walden TB, Shabalina IG, Timmons JA, Cannon B, Nedergaard J. Chronic peroxisome proliferator-activated receptor gamma (PPARgamma) activation of epididymally derived white adipocyte cultures reveals a population of thermogenically competent, UCP1-containing adipocytes molecularly distinct from classic brown adipocytes. J Biol Chem. 2010;285(10):7153-64. doi:10. 1074/jbc.M109.053942.

27. Fukui Y, Masui S, Osada S, Umesono K, Motojima K. A new thiazolidinedione, NC-2100, which is a weak PPAR-gamma activator, exhibits potent antidiabetic effects and induces uncoupling protein 1 in white adipose tissue of KKAy obese mice. Diabetes. 2000;49(5):759-67.

28. Sell H, Berger JP, Samson P, Castriota G, Lalonde J, Deshaies Y, et al. Peroxisome proliferator-activated receptor gamma agonism increases the capacity for sympathetically mediated thermogenesis 
in lean and ob/ob mice. Endocrinology. 2004;145(8):3925-34. doi: 10.1210/en.2004-0321.

29. Harms M, Seale P. Brown and beige fat: development, function and therapeutic potential. Nat Med. 2013;19(10):1252-63. doi:10.1038/ nm.3361.

30. Lanza IR, Short DK, Short KR, Raghavakaimal S, Basu R, Joyner $\mathrm{MJ}$, et al. Endurance exercise as a countermeasure for aging. Diabetes. 2008;57(11):2933-42.

31. Baar K, Wende AR, Jones TE, Marison M, Nolte LA, Chen M, et al. Adaptations of skeletal muscle to exercise: rapid increase in the transcriptional coactivator PGC-1. FASEB J. 2002;16(14): 1879-86. doi:10.1096/fj.02-0367com.

32. Handschin C, Spiegelman BM. Peroxisome proliferator-activated receptor gamma coactivator 1 coactivators, energy homeostasis, and metabolism. Endocr Rev. 2006;27(7):728-35. doi:10.1210/er. 2006-0037.

33. Kelly DP, Scarpulla RC. Transcriptional regulatory circuits controlling mitochondrial biogenesis and function. Genes Dev. 2004;18(4):357-68. doi:10.1101/gad.1177604.

34. Ruas JL, White JP, Rao RR, Kleiner S, Brannan KT, Harrison BC, et al. A PGC-1alpha isoform induced by resistance training regulates skeletal muscle hypertrophy. Cell. 2012;151(6):1319-31. doi: 10.1016/j.cell.2012.10.050.

35. Wenz T, Rossi SG, Rotundo RL, Spiegelman BM, Moraes CT. Increased muscle PGC-1alpha expression protects from sarcopenia and metabolic disease during aging. Proc Natl Acad Sci U S A. 2009;106(48):20405-10. doi:10.1073/pnas.0911570106.

36. Da Cruz S, Parone PA, Lopes VS, Lillo C, McAlonis-Downes M, Lee SK, et al. Elevated PGC-1alpha activity sustains mitochondrial biogenesis and muscle function without extending survival in a mouse model of inherited ALS. Cell Metab. 2012;15(5):778-86. doi:10.1016/j.cmet.2012.03.019.

37. Pedersen BK, Febbraio MA. Muscle as an endocrine organ: focus on muscle-derived interleukin-6. Physiol Rev. 2008;88(4):1379406. doi:10.1152/physrev.90100.2007.

38.• Erickson HP. Irisin and FNDC5 in retrospect: an exercise hormone or a transmembrane receptor? Adipocyte. 2013;2(4):289-93. doi: 10.4161/adip.26082. Paper describes lack of validity of currently available antibodies and ELISA assays for detecting irisin. Paper also discusses the limitations of using recombinant-FNDC5 (a truncated isoform of full-length FNDC5) for stimulating browning of white adiopocytes.

39. Ferrer-Martinez A, Ruiz-Lozano P, Chien KR. Mouse PeP: a novel peroxisomal protein linked to myoblast differentiation and development. Dev Dyn: Off Publ Am Assoc Anatomists. 2002;224(2): 154-67. doi:10.1002/dvdy.10099.

40. Teufel A, Malik N, Mukhopadhyay M, Westphal H. Frcp1 and Frcp2, two novel fibronectin type III repeat containing genes. Gene. 2002;297(1-2):79-83.

41.• Raschke S, Elsen M, Gassenhuber H, Sommerfeld M, Schwahn U, Brockmann B, et al. Evidence against a beneficial effect of irisin in humans. PloS one. 2013;8(9):e73680. doi:10.1371/journal.pone. 0073680. Paper demonstrates that the human sequence of FNDC5 has a reduced translational efficiency that leads to the translation of only $1 \%$ of full-length FNDC5. Furthermore, they demosntrate that the translated gene product results in a truncated isoform of FNDC5 that is is lacking the signaling peptide of irisin. Finally, they demonstrat that neither recombinant-FNDC5 nor recombinantirisin affect the browning of white adipocytes from humans.

42.• Timmons JA, Baar K, Davidsen PK, Atherton PJ. Is irisin a human exercise gene? Nature. 2012;488(7413):E9-10; discussion E-1. doi: 10.1038/nature11364. Paper demonstrates for the first time that neither endurance training nor resistance training increases skeletal muscle FNDC5 mRNA expression in healthy adults.

43. Hecksteden A, Wegmann M, Steffen A, Kraushaar J, Morsch A, Ruppenthal S, et al. Irisin and exercise training in humans - results from a randomized controlled training trial. BMC Med. 2013;11(1): 235. doi:10.1186/1741-7015-11-235. Paper reports result of a recently published randomized clinical trial that demonstrates that neither endurance training nor resistance training increase circulating irisin concentrations after 26 weeks of training compared to controls. An important observation from this study was that irisin is prone to storage-related degradation. Therefore, time related changes in circulating irisin concentrations in the absence of timed-matched controls should be interpreted with caution.

44. Pekkala S, Wiklund PK, Hulmi JJ, Ahtiainen JP, Horttanainen M, Pollanen E, et al. Are skeletal muscle FNDC5 gene expression and irisin release regulated by exercise and related to health? J Physiol. 2013;591(Pt 21):5393-400. doi:10.1113/jphysiol.2013.263707. Paper demonstrated that an acute bout of endurance exercise, chronic endurance exercise, and chronic endurance combined with resistance exercise provide conflicting results with respect to skeletal muscle PGC1- $\alpha$ expression, FNDC5 expression, and circulating irisin.

45.• Norheim F, Langleite TM, Hjorth M, Holen T, Kielland A, Stadheim HK et al. The effects of acute and chronic exercise on PGC-1alpha, irisin and browning of subcutaneous adipose tissue in human. FEBS J. 2013. doi:10.1111/febs.12619. Paper reports that twelve weeks of exercise training has little to no effect on genes expression in subcutaneous WAT for genes associated with browning of WAT (e.g., UCP1, PRDM16, TBX1, TMEM26, or CD137) in humans, despite significant increases in skeletal muscle FNDC5 gene expression.

46. Ivanov IP, Firth AE, Michel AM, Atkins JF, Baranov PV. Identification of evolutionarily conserved non-AUG-initiated Nterminal extensions in human coding sequences. Nucleic Acids Res. 2011;39(10):4220-34. doi:10.1093/nar/gkr007.

47. Roca-Rivada A, Castelao C, Senin LL, Landrove MO, Baltar J, Belen Crujeiras A, et al. FNDC5/irisin is not only a myokine but also an adipokine. PloS one. 2013;8(4):e60563. doi:10.1371/ journal.pone.0060563.

48.• Gerhard GS, Styer AM, Strodel WE, Roesch SL, Yavorek A, Carey DJ et al. Gene expression profiling in subcutaneous, visceral and epigastric adipose tissues of patients with extreme obesity. Int $\mathbf{J}$ Obes (Lond). 2013. doi:10.1038/ijo.2013.152. Paper demonstrates that FNDC5 is highly expressed in visceral adipose tissue, epigastric adipose tissue, and to a lesser extent subcutaneous adipose tissue of severely obese patients undergoing bariatric surgery. PGC1- $\alpha$ was also abundantly expressed in these adipose tissue depots. Taken together, it appears that human WAT has some of the key components necessary for FNDC5-induced browning WAT in an autocrine fashion in humans.

49. Moreno-Navarrete JM, Ortega F, Serrano M, Guerra E, Pardo G, Tinahones $\mathrm{F}$, et al. Irisin is expressed and produced by human muscle and adipose tissue in association with obesity and insulin resistance. J Clin Endocrinol Metab. 2013;98(4):E769-78. doi:10. 1210/jc.2012-2749.

50. Huh JY, Panagiotou G, Mougios V, Brinkoetter M, Vamvini MT, Schneider BE, et al. FNDC5 and irisin in humans: I. Predictors of circulating concentrations in serum and plasma and II. mRNA expression and circulating concentrations in response to weight loss and exercise. Metabolism. 2012;61(12):1725-38. doi:10.1016/j. metabol.2012.09.002. 